\title{
Activated Factor X Signaling Pathway via Protease-Activated Receptor 2 Is a Novel Therapeutic Target for Preventing Atrial Fibrillation
}

Tomomi Matsuura, MD; Takeshi Soeki, MD, PhD; Daiju Fukuda, MD, PhD; Etsuko Uematsu, PhD; Takeshi Tobiume, MD; Tomoya Hara, MD, PhD; Kenya Kusunose, MD, PhD; Takayuki Ise, MD, PhD; Koji Yamaguchi, MD, PhD; Shusuke Yagi, MD, PhD; Hirotsugu Yamada, MD, PhD; Tetsuzo Wakatsuki, MD, PhD; Masataka Sata, MD, PhD

Background: Activated factor X ( $\mathrm{FXa}$ ), which contributes to chronic inflammation via protease-activated receptor 2 (PAR2), might play an important role in atrial fibrillation (AF) arrhythmogenesis. This study aimed to assess whether PAR2 signaling contributes to AF arrhythmogenesis and whether rivaroxaban ameliorates atrial inflammation and prevents AF.

Methods and Results: In Study 1, PAR2 deficient (PAR2-/-) and wild-type mice were infused with angiotensin II (Ang II) or a vehicle via an osmotic minipump for 2 weeks. In Study 2, spontaneously hypertensive rats (SHRs) were treated with rivaroxaban, warfarin, or vehicle for 2 weeks after $8 \mathrm{~h}$ of right atrial rapid pacing. The AF inducibility and atrial remodeling in both studies were examined. Ang II-treated PAR2-I- mice had a lower incidence of AF and less mRNA expression of collagen1 and collagen3 in the atrium compared to wild-type mice treated with Ang II. Rivaroxaban significantly reduced AF inducibility compared with warfarin or vehicle. In SHRs treated with a vehicle, rapid atrial pacing promoted gene expression of inflammatory and fibrosis-related biomarkers in the atrium. Rivaroxaban, but not warfarin, significantly reduced expression levels of these genes.

Conclusions: The FXa-PAR2 signaling pathway might contribute to AF arrhythmogenesis associated with atrial inflammation. A direct FXa inhibitor, rivaroxaban, could prevent atrial inflammation and reduce AF inducibility, probably by inhibiting the proinflammatory activation.

Key Words: Anticoagulants; Atrial fibrillation; Inflammation; PAR2

A trial fibrillation (AF) is the most common type of arrhythmia encountered in clinical practice. It impairs quality of life and increases morbidity and mortality.1,2 Previous studies have suggested that inflammation contributes to the pathogenesis of AF. ${ }^{3,4}$

Accumulating evidence suggests that several coagulation proteases, including activated factor X (FXa), play an important role not only in the coagulation cascade, but also in pro-inflammatory responses through protease-activated receptors (PARs) in many cell types such as endothelial cells, platelets, fibroblasts, and smooth muscle cells., ${ }^{\mathbf{5 , 6}}$ PARs constitute a family of $\mathrm{G}$ protein-coupled, 7 transmembrane domain receptors. The PAR family comprises 4 members (PAR1 to PAR4) that have a proteolytic cleavage-based activation mechanism. ${ }^{7}$ Previous studies have

\begin{abstract}
Editorial p 1392
suggested that FXa or its major receptor, PAR2, plays an important role in the pathophysiology of inflammatory diseases, ${ }^{\mathbf{8} 10}$ including insulin resistance ${ }^{11}$ and restenosis after balloon angioplasty. ${ }^{12}$ In addition, a previous report demonstrated that cardiomyocyte-specific overexpression of PAR2 led to pathologic cardiac hypertrophy associated with cardiac fibrosis. ${ }^{13}$ Furthermore, we have recently shown that a PAR2 deletion attenuates vascular inflammation and atherogenesis in apolipoprotein E-deficient (ApoE-/-) mice. ${ }^{14}$ Direct FXa inhibitor therapy has been reported to have both anti-inflammatory and anticoagulant effects. ${ }^{15}$ We have recently reported that rivaroxaban attenuates
\end{abstract}

Received October 1, 2020; revised manuscript received January 12, 2021; accepted January 19, 2021; J-STAGE Advance Publication released online March 20, 2021 Time for primary review: 13 days

Department of Cardiovascular Medicine (T.M., T.S., E.U., T.T., K.K., T.I., K.Y., S.Y., H.Y., T.W., M.S.), Department of Community Medicine and Medical Science (T.S.), Department of Cardio-Diabetes Medicine (D.F.), Department of Community Medicine for Cardiology (H.Y.), Tokushima University Graduate School of Biomedical Sciences, Tokushima; Department of Cardiovascular Medicine, Shikoku Medical Center for Children and Adults, Zentsuji (T.H.), Japan

Mailing address: Takeshi Soeki, MD, PhD, Department of Cardiovascular Medicine, Tokushima University Graduate School of Biomedical Sciences, 2-50-1 Kuramoto, Tokushima 770-8503, Japan. E-mail: soeki@tokushima-u.ac.jp

All rights are reserved to the Japanese Circulation Society. For permissions, please e-mail: cj@j-circ.or.jp

ISSN-1346-9843 
atherosclerotic plaque progression and destabilization in apolipoprotein E-knockout mice. ${ }^{\mathbf{1 6}}$

However, it remains unknown whether PAR2-related inflammation leads to atrial remodeling and subsequent AF. In this study, we assessed whether FXa-PAR2 signaling contributes to AF arrhythmogenesis and whether rivaroxaban ameliorates atrial inflammation and prevents AF.

\section{Methods}

\section{Study 1}

Animals and Drug Administration All experimental procedures were performed according to the guidelines for animal experimentation of the University of Tokushima. For Study 1, PAR2-deficient mice (PAR2-/-, C57BL/6J background) were originally purchased from Jackson Laboratory (California, USA). Eight-week-old male CL57/ B6 mice were purchased from Japan SLC (Hamamatsu, Japan). Eight-week-old male PAR2-/- mice $(n=12)$ and wild-type $(\mathrm{WT})$ mice $(\mathrm{n}=14)$ were infused with angiotensin II (Ang II) $(2 \mathrm{mg} / \mathrm{kg} /$ day $)$ or a vehicle via an osmotic minipump (ALZET model 2004; DURECT, Cupertino, CA, USA) for 2 weeks. We measured blood pressure with the tail-cuff method before and every week after the Ang II or a vehicle infusion and performed transthoracic echocardiography. Next, we examined the inducibility of AF in an intracardiac electrophysiological study and inflammationinduced atrial remodeling with a biochemical analysis. This study was approved by the Committee for Animal Experiments of Tokushima University (Reference number: T30-137) and the genetic modification experiment safety management Committee of Tokushima University (Reference number: 27-67).

AF Induction We examined the effects of PAR2 on AF inducibility in an intracardiac electrophysiological study with rapid pacing from the right atrium. The pacing pulse used for the induction of AF was rectangular in shape and $5 \mathrm{~V}$ (approximately 3-fold the diastolic threshold voltage) with a 6-ms width. Burst right atrial stimulation with a cycle length of $20 \mathrm{~ms}$ for $30 \mathrm{~s}$ via the distal electrode pair of the catheter was performed using an electrical stimulator to induce AF. Burst pacing and AF measurement were repeated 3-fold, with a 5-min break in between. The induced rhythm was defined as AF when a fast atrial irregular rhythm was maintained for more than $2 \mathrm{~s}$.

Histology and Real-Time Polymerase Chain Reaction (PCR) Each heart was cut along a horizontal plane between the lower tips of the left and right atria. The upper portion was snap-frozen in an optimal cutting temperature compound. Next, the atrium was sectioned serially (5- $\mu \mathrm{m}$ intervals). The lower portion was used for assessing gene expression of markers related to inflammation and fibrosis.

We evaluated the degree of fibrosis using Masson's trichrome staining. We also evaluated markers for activated form fibroblasts using anti-periostin antibody (ab14041; Abcam, Cambridge, UK) and indicator for reactive oxygen species (ROS) using anti-nicotinamide adenine dinucleotide phosphate oxidase 4 (NOX4) antibody (14347-1-AP; Proteintech, Rosemont, IL, USA), followed by immunohistochemical staining with the peroxidase-anti-peroxidase technique. Total RNA was extracted from tissues and cells using NucleoSpin RNA (MACHEREY-NAGEL GmbH \& Co. KG., Dueren, Germany). Reverse transcription was performed using a QuantiTect Reverse Transcription kit (QIAGEN, Hilden, Germany) with $1 \mu \mathrm{g}$ of total extracted
RNA. Quantitative real-time PCR was performed on an Mx3000P (Agilent Technologies, CA, USA) using genespecific primers and a Power SYBR Green PCR Master Mix (Applied Biosystems, CA, USA). Data are expressed in arbitrary units normalized by Glyceraldehyde 3-phosphate dehydrogenase.

\section{Statistical Analysis}

Numerical values are expressed as means \pm standard error of the mean (SEM). Comparison of parameters between the 2 groups was performed with the unpaired Student's t-test. Differences between multiple groups were analyzed using 1-way analysis of variance, followed by Dunnett's post-hoc analysis. $\mathrm{P}<0.05$ was considered significant.

\section{Study 2}

Animals and Drug Administration In Study 2, spontaneously hypertensive rats (SHRs) were purchased from Japan SLC (Hamamatsu, Japan). Rapid atrial stimulation at a frequency of 1,200 beats/min with $0.1-\mathrm{ms}$ rectangular pulses was applied to the right atrium of 8-week-old male SHRs $(n=45)$ for $8 \mathrm{~h}$ to induce electrical and histological remodeling using a programmable stimulator. Next, the rats were divided into 3 groups: a group treated with rivaroxaban $(2,400 \mathrm{mg} / \mathrm{kg}$ of diet/day, orally), a group treated with warfarin $(0.2 \mathrm{mg} / \mathrm{kg}$ body weight/day, orally), and a group treated with a vehicle. Treatment was given for 2 weeks. Rivaroxaban was supplied by Bayer HealthCare AG (Berlin, Germany). This study was approved by the Committee for Animal Experiments of Tokushima University (Reference number: T30-137) and the genetic modification experiment safety management Committee of Tokushima University (Reference number: 27-67).

AF Induction We examined the effects of rivaroxaban on AF inducibility in an intracardiac electrophysiological study that included rapid pacing from the right atrium. The pacing pulse used for the induction of AF was rectangular in shape and $10 \mathrm{~V}$ (approximately 3-fold the diastolic threshold voltage) with a $6 \mathrm{~ms}$ width. Burst right atrial stimulation with a cycle length of $12 \mathrm{~ms}$ for $30 \mathrm{~s}$ via the distal electrode pair of the catheter was performed to induce AF using an electrical stimulator. Burst pacing and AF measurement were repeated 3-fold, with a 5-min break in between. The induced rhythm was defined as AF when a fast atrial irregular rhythm was maintained for more than $2 \mathrm{~s}$.

Histology and Real-Time PCR We used the same methods used in Study 1. However, after 2 weeks of treatment, Masson's trichrome staining of the left atrium showed extremely mild fibrosis and differences among groups were not significant (data not shown). Therefore, we evaluated the degree of fibrosis in each group after 3 months of treatment. We used age-matched male normotensive SpragueDawley (SD) rats as control ( $\mathrm{n}=5$ rats per group).

\section{Statistical Analysis}

We used the same method used in Study 1.

\section{Results}

\section{Study 1}

Physiological and Echocardiographic Characteristics of the Mice Table 1 shows the physiological and echocardiographic characteristics of the mice. There were no significant differences in body weight, heart-to-body weight 
Table 1. Physiological and Echocardiographic Characteristics of the Mice (Study 1)

\begin{tabular}{lccccc} 
& $\begin{array}{c}\text { WT-Vehicle } \\
(\mathbf{n}=7)\end{array}$ & $\begin{array}{c}\text { PAR2-/- }- \text { Vehicle } \\
(\mathbf{n}=6)\end{array}$ & $\begin{array}{c}\text { WT-Ang II } \\
(\mathbf{n}=\mathbf{7})\end{array}$ & $\begin{array}{c}\text { PAR2-/- -Ang II } \\
(\mathbf{n}=6)\end{array}$ & P value \\
Body weight $(\mathrm{g})$ & $25 \pm 1$ & $29 \pm 3$ & $24 \pm 1$ & $28 \pm 3$ & NS \\
Heart/body weight ratio $(\mathrm{g} / \mathrm{g})$ & 0.004 & 0.005 & 0.005 & 0.006 & NS \\
SBP $(\mathrm{mmHg})$ & $121 \pm 5$ & $112 \pm 6$ & $154 \pm 8^{*}$ & $138 \pm 8^{* *}$ & \\
Heart rate (beats/min) & $568 \pm 25$ & $567 \pm 49$ & $652 \pm 38$ & $659 \pm 36$ & NS \\
LVDd (mm) & $2.7 \pm 0.2$ & $3.0 \pm 0.3$ & $2.9 \pm 0.1$ & $2.8 \pm 0.1$ & NS \\
FS (\%) & $47.0 \pm 2.6$ & $47.7 \pm 2.9$ & $48.8 \pm 2.7$ & $49.2 \pm 2.0$ & NS \\
\hline
\end{tabular}

${ }^{*} \mathrm{P}<0.01$ vs. WT-Vehicle, PAR2-/- -Vehicle. ${ }^{* *} \mathrm{P}<0.05$ vs. PAR2-/- -Vehicle. Values are presented as means $\pm S E M$. Ang II, angiotensin II; FS, fractional shortening; PAR, protease-activated receptor; LVDd, left ventricular end-diastolic dimension; NS, not significant; SBP, systolic blood pressure; WT, wild type.

A

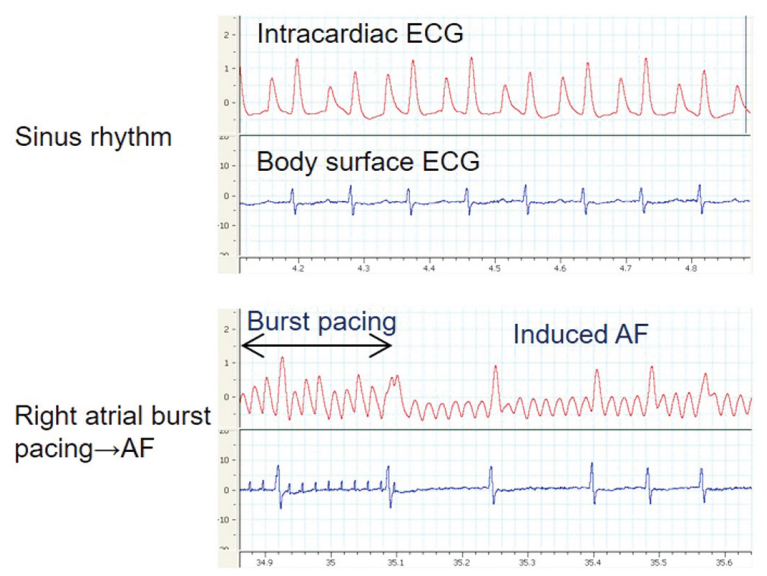

B

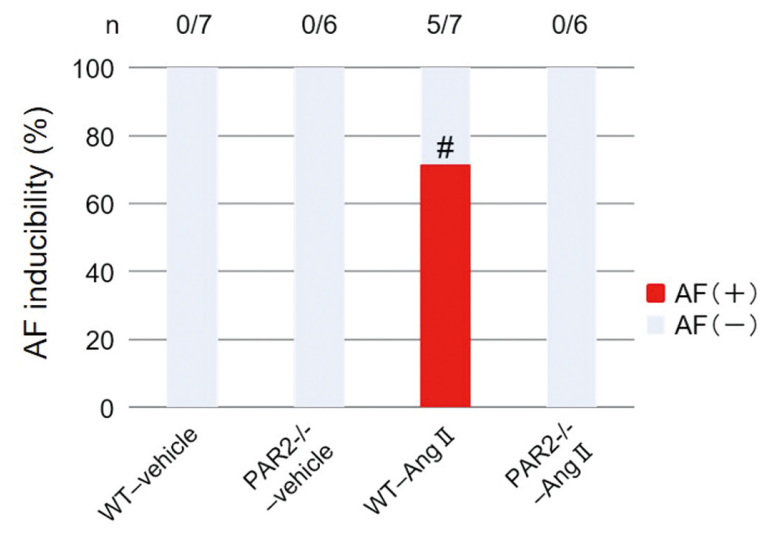

Figure 1. Representative example of atrial fibrillation (AF) induced in a mouse (Study 1). (A) Representative body-surface and intracardiac electrocardiogram (ECG) recordings before and after right atrial burst pacing. Burst pacing was followed by the induction of AF. (B) Incidence of AF. Burst pacing induced AF in 0 of $7(0 \%)$ wild-type (WT)-vehicle mice and angiotensin II (Ang II) infusion increased the induction ratio in 5 of 7 (71\%) rats. No AF was induced in protease-activated receptor 2 deficient (PAR2-/-) mice with or without Ang II infusion (PAR2-/- -vehicle 0/6, PAR2-/- -Ang II 0/6). \#P<0.05 vs. WT-vehicle group, PAR2-/- -vehicle group and PAR2-/- -Ang II group.

ratio, and heart rate between the 4 groups. Echocardiography revealed no significant differences in left ventricular end-diastolic diameter (LVDd) and \% fractional shortening $(\% \mathrm{FS})$ between the 4 groups (Table 1). Before Ang II infusion, there were no significant differences in systolic blood pressure between wild type (WT) and PAR2-/mice. A continuous Ang II infusion caused an elevation in systolic blood pressure and heart rate in WT-Ang II and PAR2-/- -Ang II mice. However, there were no significant differences in these parameters between WT and PAR2-/- mice with and without Ang II infusion.

Induction of AF With Right Atrial Burst Pacing Representative body-surface and intracardiac electrocardiogram recordings before and after right atrial burst pacing are presented in Figure 1A. In Figure 1A, burst pacing was followed by the induction of AF. Burst pacing induced AF in 0 of $7(0 \%)$ WT-vehicle mice. Ang II infusion increased the induction ratio to $5 / 7(71 \%)$. Interestingly, AF was not induced in PAR2-/- mice with or without Ang II infusion (PAR2-/- -vehicle, 0/6; PAR2-/- -Ang II, 0/6) (Figure 1B).

Collagen Volume Fraction, Gene Expression and Immuno- histochemistry in the Atrium Figure 2A shows the representative results of Masson's trichrome staining of the left atrium. The quantitative ratio of the area of fibrosis to the area of reference tissue is summarized in Figure 2B. Masson's trichrome staining revealed marked heterogeneous fibrosis in the left atrium of WT-Ang II mice. There was less interstitial fibrosis in PAR2-/- -Ang II mice compared with WT-Ang II mice.

Atrial gene expression is presented in Figure 3. PAR1 $[F 2 r]$ mRNA expression, but not PAR2 [F2rll] expression, was detected in PAR2-/- mice. Collagen1 [Collal] and collagen3 [Col3al] mRNA expression levels were remarkably higher in WT-Ang II mice than in mice that did not receive Ang II infusion. Increased mRNA expression of Collal and Col3al was attenuated in PAR2-/- mice.

We also evaluated the expression of activated form fibroblasts using anti-periostin antibody (Supplementary Figure 1). Although increased staining of periostin was seen in the left atrium of WT-Ang II mice, it was not attenuated in PAR2-/- -Ang II mice (Supplementary Figure 1A,B). The mRNA level of periostin [Postn] was 


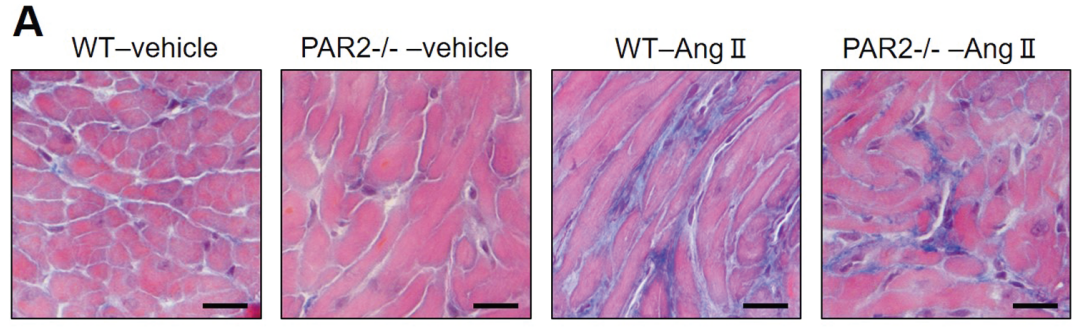

B

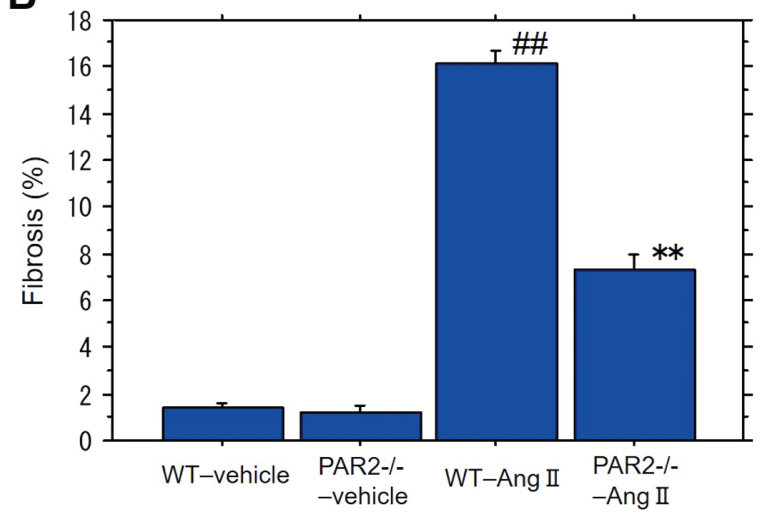

Figure 2. Histological findings in the left atrium (Study 1). (A) Representative results of Masson's trichrome staining of the left atrium. Blue staining indicates interstitial fibrosis. Scale bar= $20 \mu \mathrm{m}$. (B) Quantitative ratio of the area of fibrosis to the area of reference tissue is shown. Masson's trichrome staining revealed remarkable heterogeneous fibrosis in the left atrium of the wild-type (WT)-angiotensin II (Ang II) mice. There was less interstitial fibrosis in the protease-activated receptor 2 deficient (PAR2-/-) -Ang II mice compared to the WT-Ang II mice. ${ }^{* *} \mathrm{P}<0.01$ vs. WT-vehicle group and PAR2-/- -vehicle group. \#P<0.01 vs. PAR2-/- -Ang II group, WT-vehicle group, and PAR2-/- -vehicle group.
A

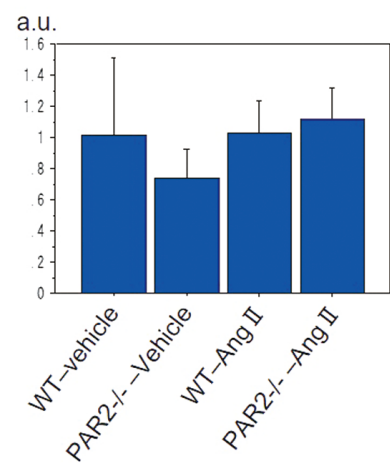

B

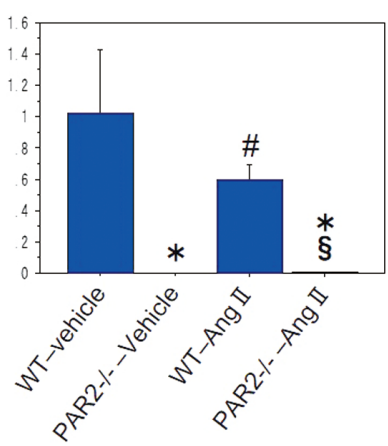

C $\quad \underline{\text { Col1a1 }}$

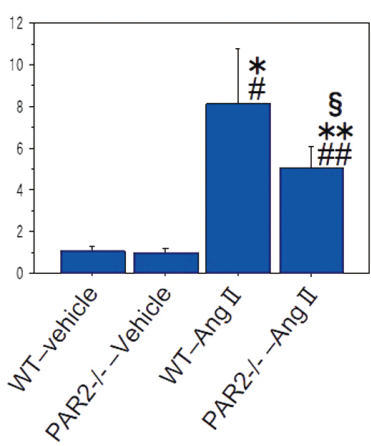

D $\underline{\text { Col3a1 }}$

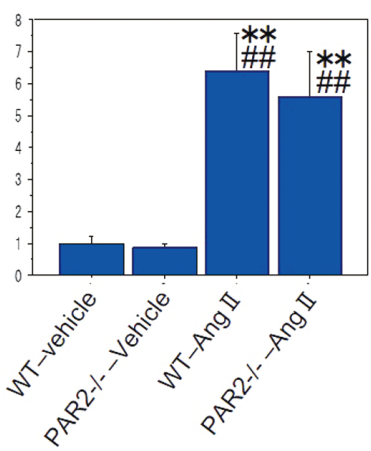

Figure 3. Gene expression in the atrium (Study 1). (A,B) In protease-activated receptor 2 deficient (PAR2-/-) mice, mRNA expression of protease-activated receptor (PAR)1 (F2r), but not PAR2 (F2rl1), was detected. (C,D) Compared to mRNA expression in mice who did not receive angiotensin II (Ang II), mRNA expression of collagen1 (Col1a1) and collagen3 (Col3a1) was remarkably higher in wildtype (WT)-Ang II mice. The higher levels of Col1a1 and Col3a1 mRNA expression were attenuated in PAR2-/- mice. ${ }^{\star *} \mathrm{P}<0.01$ vs. WT-vehicle group. ${ }^{*} \mathrm{P}<0.05$ vs. WT-vehicle group. ${ }^{\#} \mathrm{P}<0.01$ vs. PAR2 $-/--$ vehicle group. ${ }^{\mathrm{P}}<0.05$ vs. PAR2 $-/--$ vehicle group. $\$ \mathrm{P}<0.05 \mathrm{vs}$. WT-Ang II group.

similar to the results of its immunohistochemistry (Supplementary Figure 1C). Furthermore, we evaluated the expression of the ROS-generating enzyme, NOX4, using anti-NOX4 antibody (Supplementary Figure 1D,E). Increased staining of NOX4 was seen in the left atrium of WT-Ang II mice; it was attenuated in PAR2-/- -Ang II mice.

\section{Study 2}

Physiological and Echocardiographic Characteristics of the

Rats SHRs treated with rivaroxaban had lower body weight than the other 2 groups. Echocardiography revealed no significant differences in $\% \mathrm{FS}$ between the 3 groups. SHRs treated with rivaroxaban had a smaller LVDd than SHRs treated with vehicle. There were no significant differences in the heart-to-body weight ratio, systolic blood pressure, heart rate, and other echocardiographic characteristics between SHRs treated with vehicle, warfarin, and rivaroxaban (Table 2).

Induction of AF With Right Atrial Burst Pacing Right atrial burst pacing induced AF in 14 of $15(93 \%)$ SHRs 


\begin{tabular}{|c|c|c|c|c|}
\hline & $\begin{array}{c}\text { Vehicle } \\
(n=15)\end{array}$ & $\begin{array}{c}\text { Warfarin } \\
(n=15)\end{array}$ & $\begin{array}{l}\text { Rivaroxaban } \\
\quad(n=15)\end{array}$ & $P$ value \\
\hline Body weight (g) & $231 \pm 8^{*}$ & $226 \pm 8^{*}$ & $214 \pm 11$ & \\
\hline Heart-to-body weight ratio $(\mathrm{g} / \mathrm{g})$ & 0.004 & 0.004 & 0.004 & NS \\
\hline $\mathrm{SBP}(\mathrm{mmHg})$ & $154 \pm 17$ & $155 \pm 12$ & $153 \pm 16$ & NS \\
\hline Heart rate (beats/min) & $405 \pm 46$ & $395 \pm 35$ & $391 \pm 30$ & NS \\
\hline LVDd (mm) & $6.85 \pm 0.60$ & $6.76 \pm 0.45$ & $6.43 \pm 0.51^{\star *}$ & \\
\hline FS (\%) & $49.12 \pm 2.36$ & $49.08 \pm 1.38$ & $48.13 \pm 2.91$ & NS \\
\hline
\end{tabular}

${ }^{*} \mathrm{P}<0.01$ vs. rivaroxaban. ${ }^{* \star} \mathrm{P}<0.01$ vs. vehicle. Values are presented as means $\pm S E M$. FS, fractional shortening; LVDd, left ventricular end-diastolic dimension.

treated with vehicle. The administration of warfarin did not significantly change the AF induction ratio (12/15, $80 \%$ ) compared to the ratio with vehicle. However, rivaroxaban was associated with significantly lower AF inducibility $(5 / 15,33 \%)$ compared with warfarin or vehicle (Figure 4).

Atrial Gene Expression and Immunohistochemistry Figure 5 shows atrial gene expression. In SHRs treated with vehicle, rapid pacing promoted the gene expression of tumor necrosis factor- $\alpha[T n f]$, monocyte chemoattractant protein-1 [ $C c l 2]$, and transforming growth factor $\beta$ [ $T g f b 2]$ in the atrium. Rivaroxaban, but not warfarin, significantly reduced expression levels of these genes. Rapid pacing also promoted the gene expression of Collal and Col3al in the atrium. Rivaroxaban significantly reduced expression of these genes. In addition, rivaroxaban also significantly reduced gene expression of $F 2 r$ and $F 2 r l l$, the major receptors for thrombin and factor Xa.

Increased staining of periostin, a marker for activated fibroblasts, was seen in the left atrium of SHR-vehicle and SHR-warfarin groups; that was remarkably attenuated in the SHR-rivaroxaban group (Supplementary Figure 2A,B). In addition, the mRNA level of periostin [Postn] was similar to the results of its immunohistochemistry (Supplementary Figure 2C). Increased staining of NOX4, ROS-generating enzyme, was seen in the left atrium of SHR-vehicle and SHR-warfarin groups; that was attenuated in the SHRrivaroxaban group (Supplementary Figure 2D,E).

Collagen Volume Fraction in the Atrium The quantitative ratio of the area of fibrosis to the area of reference tissue after 3 months of treatment is summarized in Figure 6. Masson's trichrome staining revealed heterogeneous fibrosis in the left atrium of the SHR-vehicle and SHR-warfarin groups. Interstitial fibrosis was attenuated in the SHR-rivaroxaban group. A few fibrotic foci were observed in the age-matched male control rat group.

\section{Discussion}

This study demonstrated that Ang II-treated PAR2-/mice had a lower incidence of AF, less fibrotic change, and lower mRNA expression of Collal and Col3al in the atrium compared with WT mice treated with Ang II. It also showed that the administration of rivaroxaban significantly reduces AF inducibility and gene expression of inflammatory and fibrosis-related biomarkers in the atrium compared with administration of warfarin or vehicle.

Accumulating evidence suggests that the FXa-PARs pathway promotes pro-inflammatory responses in many cell types, contributing to the pathogenesis of inflammatory diseases. ${ }^{\mathbf{8}-12}$ However, little is known about the rela-

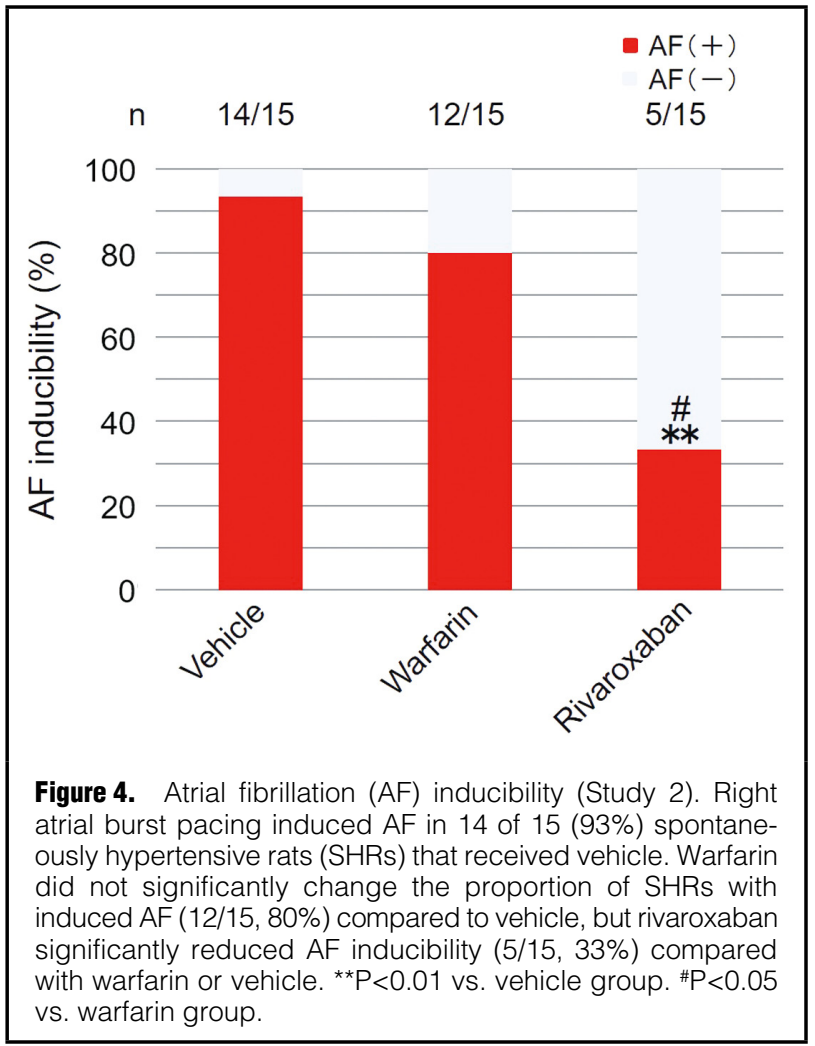

tionship between the FXa-PAR2 pathway and AF arrhythmogenesis. In this study, we found evidence that PAR2 deficiency contributes to lower AF inducibility, probably via the suppression of atrial fibrosis in PAR2-/mice. The mechanism of AF observed in Ang II-treated mice can be postulated to be reentrant because of an atrial conduction disturbance; it was associated with Ang IIinduced atrial interstitial fibrosis. Some previous studies showing that PAR2 signaling contributes to cardiovascular fibrosis ${ }^{\mathbf{1 3 , 1 7}}$ support our findings. A previous study demonstrated that PAR2 is involved in Ang II-induced aortic adventitial fibroblast activation. ${ }^{17}$ Another study reported that cardiomyocyte-specific overexpression of PAR2 results in cardiac fibrosis and enhanced expression of fibrosis-related genes such as $T g f b 2$ and Col3al. ${ }^{13}$ In addition, a previous in vitro study demonstrated that FXa enhances fibroblast proliferation and migration, at least partially via the activator protein 1 and nuclear factor- $\kappa \mathrm{B}$ pathway. ${ }^{\mathbf{1 8}}$ 

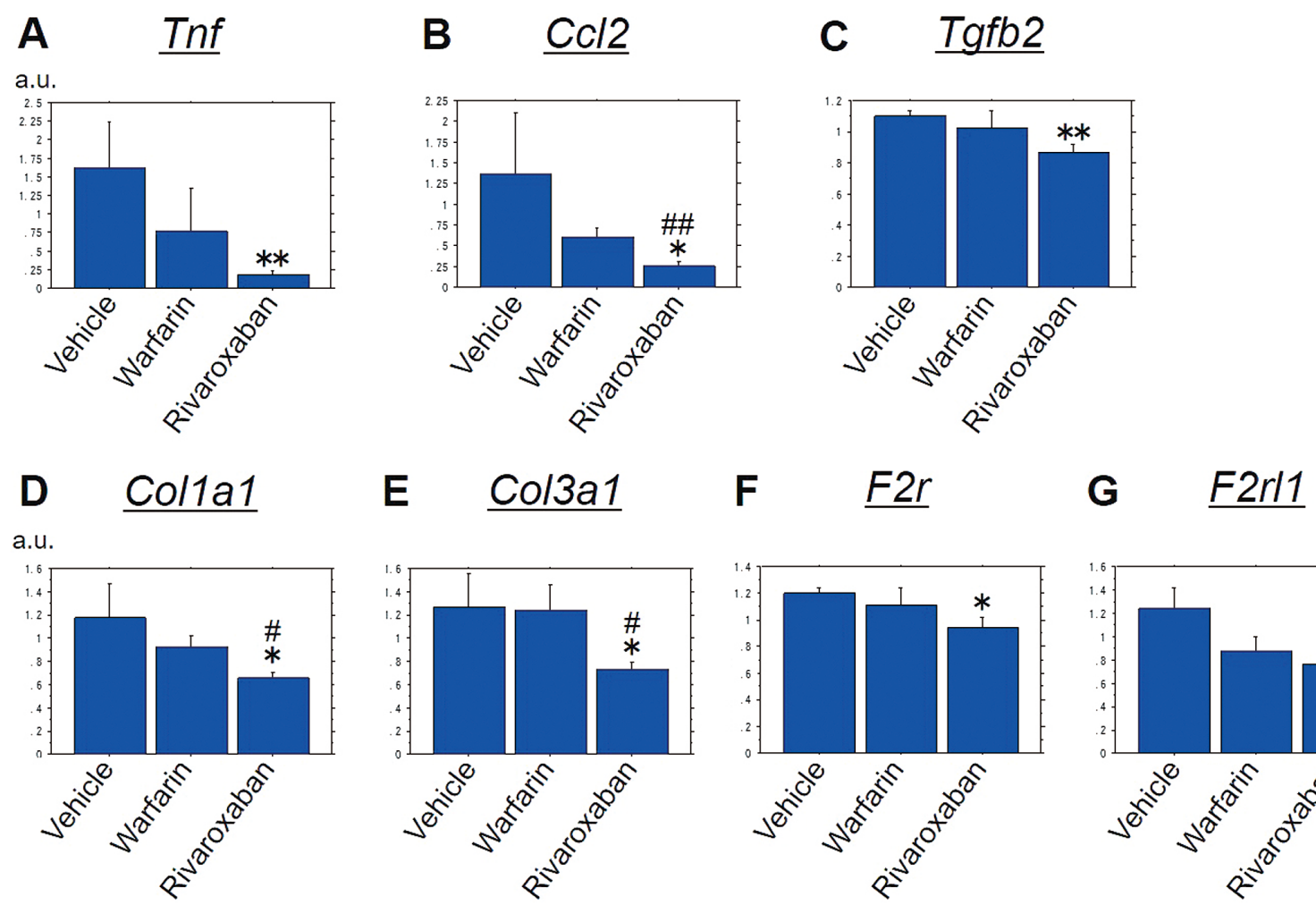

Figure 5. Gene expression in the atrium (Study 2). (A-C) In spontaneously hypertensive rats (SHRs) treated with vehicle, rapid pacing promoted gene expression of tumor necrosis factor-a (Tnf), monocyte chemoattractant protein-1 (Ccl2), and transforming growth factor- $\beta$ (Tgfb2) in the atrium. Rivaroxaban, but not warfarin, significantly reduced the expression levels of these genes. (D,E) Rapid pacing also promoted the gene expression of collagen1 (Col1a1) and collagen3 (Col3a1) in the atrium. Rivaroxaban significantly reduced expression levels of these 2 genes. $(\mathbf{F}, \mathbf{G})$ Rivaroxaban also significantly reduced the gene expression of protease-activated receptor (PAR) 1 (F2r) and PAR2 (F2r/1). ${ }^{* *} \mathrm{P}<0.01$ and ${ }^{*} \mathrm{P}<0.05$ vs. vehicle group. ${ }^{\#} \mathrm{P}<0.01$ and ${ }^{\# P}<0.05$ vs. warfarin group.

These previous findings and this study support our hypothesis that FXa-PAR2 signaling contributes to AF arrhythmogenesis, at least in part via accelerated atrial fibrosis.

In this study, we also showed that the administration of rivaroxaban significantly reduces $\mathrm{AF}$ inducibility compared with administration of warfarin or vehicle in SHRs. In addition, rivaroxaban, but not warfarin, significantly reduced gene expression of inflammatory and fibrosisrelated biomarkers. There was a similar degree of extremely mild left atrial interstitial fibrosis among the 3 groups after 2 weeks of treatment. Thus, we evaluated the degree of left atrial interstitial fibrosis after 3 months of treatment. We found that interstitial fibrosis after 3 months of treatment was attenuated in SHRs treated with rivaroxaban when compared to that in SHRs treated with warfarin or vehicle. As the progression of fibrosis in SHRs is thought to be slower than in Ang II-treated mice, a treatment period of 2 weeks might be too short for assessing histologic changes. There are few reports about whether rivaroxaban ameliorates atrial inflammation and prevents AF. A recent study reported that in patients with chronic non-valvular $\mathrm{AF}$, compared with warfarin, rivaroxaban was associated with a greater increase in thrombomodulin (TM) levels and a trend towards a reduction in matrix metalloproteinase 9 levels over 24 weeks. ${ }^{19}$ Another study also reported that plasma concentrations of pentraxin 3 decreased and those of TM increased after the initiation of oral anticoagulant therapy with FXa inhibitors in patients with non-valvular AF. ${ }^{20}$ In an in vitro study, FXa and tachyarrhythmias acted synergistically to increase the expression of PARs, the inflammatory mediators, interleukin- 8 and intercellular adhesion molecule-1, and endothelial factors such as prooxidative lectin-like oxidized LDL receptor-1 and prothrombotic plasminogen activator inhibitor-1 in human atrial tissue. Rivaroxaban effectively prevented FXainduced molecular effects in human atrial tissue, particularly during rapid pacing. ${ }^{21}$ Kondo et al reported that rivaroxaban attenuates atrial inflammatory fibrosis by inhibiting the activation and upregulation of PAR2 and reduced AF inducibility in pressure-overloaded hearts. They pointed out the possibility that rivaroxaban has the potential to prevent AF. ${ }^{22}$ Those results are compatible with our results. Differences in the 2 studies included: (1) we had a warfarin group in addition to a rivaroxaban group; (2) we examined AF inducibility in an in vivo AF model; (3) we used a model of spontaneous hypertension, which differs from the transverse aortic constriction (TAC) model; and (4) we examined the contribution of PAR2 signaling to AF in PAR2-/- mice. Therefore, we believe that the AF model in this study was more physiologic and 


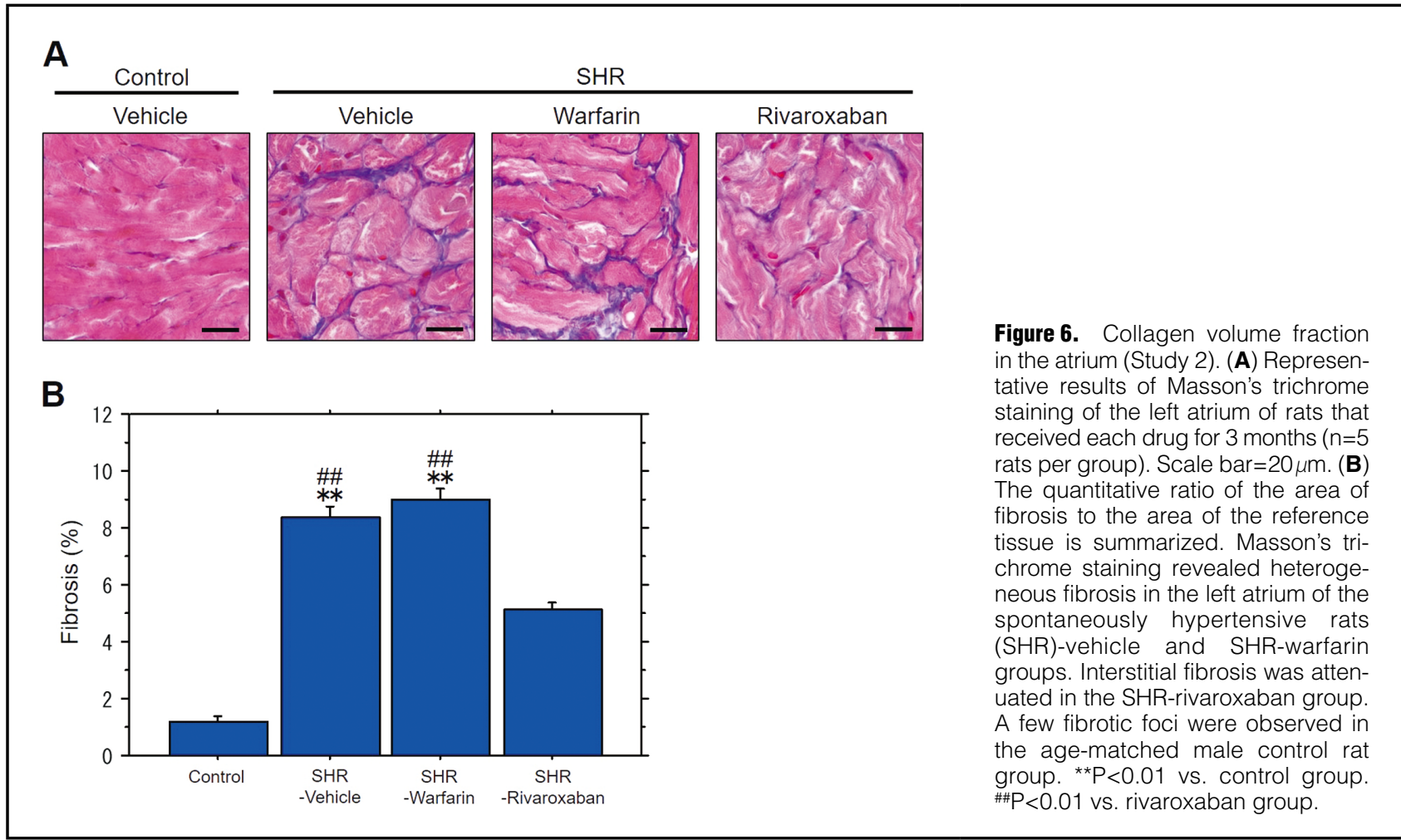

closer to practical clinical conditions than the previous study's model. ${ }^{22}$ Based on the results of our study and previous studies, we speculate that rivaroxaban might attenuate atrial remodeling, at least in part by inhibiting pro-inflammatory activation via PAR2.

Previous studies reported that PAR2 antagonists might be useful for treating various inflammatory diseases. They might also be effective in reducing AF. Chung et al reported that GB83, an antagonist of PAR2, significantly decreased migratory capability of atrial fibroblasts, procollagen type I production, and rate of human atrial fibroblast proliferation. ${ }^{23}$ Imano et al suggested that FSLLRY, another PAR2 antagonist, and rivaroxaban are able to suppress the generation of reactive oxygen species induced by intermittent hypoxia through the inhibition of PAR2 in a mouse model and consequently attenuate cardiac remodeling. ${ }^{24}$ However, there are no data about whether PAR2 antagonists prevent AF; thus, a more detailed investigation needs to be conducted. In contrast, many studies have reported that the activation of PAR2 provokes arterial and venous dilation in humans. ${ }^{25-27}$ PAR2 is highly expressed in endothelial cells and its activation causes endothelium-dependent and endothelium-independent relaxation of human vessels in pathological conditions in which inflammatory cytokines are released. ${ }^{28,29}$ Those data suggest that PAR2 plays a role in regulating systemic and local hemodynamics. In other words, PAR2 may help preserve blood supply to organs and blood pressure. PAR2 may also be protective against ischemic injury to multiple organs in diseases related to inflammatory cytokines. Therefore, we need more research about whether particular types of ischemia-related diseases can be aggravated by the use of strong PAR2 antagonists before their clinical use.

In the present study, we used 2 types of hypertension models causing inflammatory atrial remodeling. Although the TAC model and the Ang infusion model were reported as mice models of hypertension, we selected the latter for Study 1 because we think the latter model is more physiological and close to the clinical situation of hypertension. In Study 2, we used SHRs as a more clinically physiological hypertension model in rats. It is reported that the reninangiotensin-aldosterone system (RAAS) has been implicated in the pathogenesis of hypertension in SHRs. ${ }^{30}$ In addition, a previous study reported that the level of expressed mRNA of the cardiac RAAS in SHRs was higher than that in normotensive controls. ${ }^{31}$ Furthermore, it is reported that an angiotensin II antagonist prevents electrical and structural remodeling in a rapid atrial pacing dog model. ${ }^{32,33}$ These reports suggest that the renin-angiotensin system is activated in a rapid atrial pacing model. Thus, we believe that RAAS activation is at least one of the common mechanisms for 2 different types of hypertension associated with atrial inflammatory remodeling in Study 1 and Study 2.

One clinical implication of this study is that direct oral anticoagulant therapy might be effective in preventing both AF and thrombotic events in patients at high risk for AF who do not have evidence of AF. Several studies have reported that $\mathrm{CHADS}_{2}$ (congestive heart failure, hypertension, age $>75$ years, diabetes mellitus, and previous stroke/ transient ischemic attack [doubled]) and $\mathrm{CHA}_{2} \mathrm{DS}_{2}-\mathrm{VASc}$ (congestive heart failure, hypertension, age $\geq 75$ [doubled], diabetes mellitus, prior stroke or transient ischemic attack [doubled], vascular disease, age 65-74, female) scores may predict the risk of stroke or thromboembolic events in the absence of AF ${ }^{34-36}$ Furthermore, those scores have also been reported to predict new-onset AF. ${ }^{37,38}$ Taking the results of this study and previous findings into consideration, 
direct FXa inhibitors may be effective in preventing new-onset $\mathrm{AF}$ and subsequent thromboembolic events in patients with high $\mathrm{CHADS}_{2}$ or $\mathrm{CHA}_{2} \mathrm{DS}_{2}-\mathrm{VASc}$ scores who do not have AF. Furthermore, in our preliminary study, plasma concentration of rivaroxaban was slightly lower than the level of anticoagulant action in SHRs treated with the same dose of rivaroxaban used in the present study (data not shown). Therefore, we believe the low dose of rivaroxaban might be effective in preventing AF in clinical practice.

\section{Study Limitations}

There were several limitations to this study. First, we did not evaluate which cells contributed to the PAR2 signaling involved in atrial remodeling and AF; however, we hypothesize that the effect of PAR2 on fibroblasts is mainly responsible for atrial remodeling. We could not evaluate whether the effect of PAR2 on macrophages contributed to atrial inflammation and fibrosis. Second, it was not clear whether rivaroxaban has any clinical drug effects or if direct FXa inhibitors have class effects. To date, there have not been any studies comparing the ability to prevent AF inducibility across direct FXa inhibitors. Third, we did not evaluate whether the effect of rivaroxaban was dose-dependent or length-dependent. Fourth, we defined AF when a fast atrial irregular rhythm was maintained for more than $2 \mathrm{~s}$. But it is not confirmed whether this phenomenon has similar pathologic significance for human AF. Finally, we only examined the role of FXa-PAR-2 signaling in AF; however, FXa activates PAR-1 as well. Some previous studies reported the contribution of PAR-1 signaling to inflammation. ${ }^{39,40}$ It has been unclear how much the FXaPAR2 signaling pathway contributes to atrial inflammation and remodeling compared with other inflammatory signaling, including PAR-1, Ca2+ overload, and NF- $\kappa \mathrm{B}$ activation. Further studies are needed to clarify the role of other inflammatory signaling in AF.

\section{Conclusions}

The FXa-PAR2 signaling pathway might contribute to AF arrhythmogenesis associated with atrial inflammation. A direct FXa inhibitor, rivaroxaban, could prevent atrial inflammation and reduce AF inducibility, probably by inhibiting pro-inflammatory activation.

\section{Acknowledgments}

M.S. received research funding from Bayer Yakuhin. The funder had no role in study design, data collection, data analysis, or preparation of the manuscript.

This work was partially supported by JSPS Kakenhi Grants (Number 18K08077 to T.S., Number 17K09506 to K.K., Number 18K08040 to S.Y., Number 19K08584 to D.F., and Number $19 H 03654$ to M.S.) and grants from the Japanese Society of Echocardiography (to H.Y.), Takeda Science Foundation (to K.K. and M.S.), Fugaku Trust for Medical Research (to M.S.) and the Vehicle Racing Commemorative Foundation (to M.S.).

\section{Disclosures}

The authors declare no potential conflicts of interest.

\section{IRB Information}

The present study was approved by the Committee for Animal Experiments of Tokushima University (Reference number: T30-137) and the genetic modification experiment safety management Committee of Tokushima University (Reference number: 27-67).

\section{References}

1. Brand FN, Abbott RD, Kannel WB, Wolf PA. Characteristics and prognosis of lone atrial fibrillation: 30 -year follow-up in the Framingham Study. JAMA 1985; 254: 3449-3453.

2. Benjamin EJ, Wolf PA, D'Agostino RB, Silbershatz H, Kannel WB, Levy D. Impact of atrial fibrillation on the risk of death: The Framingham Heart Study. Circulation 1998; 98: 946-952.

3. Chung MK, Martin DO, Sprecher D, Wazni O, Kanderian A, Carnes CA, et al. C-reactive protein elevation in patients with atrial arrhythmias: Inflammatory mechanisms and persistence of atrial fibrillation. Circulation 2001; 104: 2886-2891.

4. Engelmann MD, Svendsen JH. Inflammation in the genesis and perpetuation of atrial fibrillation. Eur Heart J 2005; 26: 2083-2092.

5. Borissoff JI, Spronk HM, ten Cate H. The hemostatic system as a modulator of atherosclerosis. $N$ Engl J Med 2011; 364: 1746-1760.

6. Croce $\mathrm{K}$, Libby P. Intertwining of thrombosis and inflammation in atherosclerosis. Curr Opin Hematol 2007; 14: 55-61.

7. Borensztajn K, Spek CA. Blood coagulation factor Xa as an emerging drug target. Expert Opin Ther Targets 2011; 15: 341-349.

8. Borensztajn K, Peppelenbosch MP, Spek CA. Factor Xa: At the crossroads between coagulation and signaling in physiology and disease. Trends Mol Med 2008; 14: 429-440.

9. Rothmeier AS, Ruf W. Protease-activated receptor 2 signaling in inflammation. Semin Immunopathol 2012; 34: 133-149.

10. Spronk HM, de Jong AM, Crijns HJ. Pleiotropic effects of factor $\mathrm{Xa}$ and thrombin: What to expect from novel anticoagulants. Cardiovasc Res 2014; 101: 344-351.

11. Badeanlou L, Furlan-Freguia C, Yang G, Ruf W, Samad F. Tissue factor-protease-activated receptor 2 signaling promotes dietinduced obesity and adipose inflammation. Nat Med 2011; 17: $1490-1497$.

12. Ragosta M, Gimple LW, Gertz SD, Dunwiddie CT, Vlasuk GP, Haber HL, et al. Specific factor Xa inhibition reduces restenosis after balloon angioplasty of atherosclerotic femoral arteries in rabbits. Circulation 1994; 89: $1262-1271$.

13. Antoniak S, Sparkenbaugh EM, Tencati M, Rojas M, Mackman $\mathrm{N}$, Pawlinski R. Protease activated receptor-2 contributes to heart failure. PLoS One 2013; 8: e81733.

14. Hara T, Phuong PT, Fukuda D, Yamaguchi K, Murata C, Nishimoto $S$, et al. Protease-activated receptor-2 plays a critical role in vascular inflammation and atherosclerosis in apolipoprotein E-deficient mice. Circulation 2018; 138: 1706-1719.

15. Iba T, Aihara K, Watanabe S, Yanagawa Y, Yamada A, Koichiro $\mathrm{N}$, et al. Factor Xa inhibitor attenuates leukocyte adhesion and thrombus formation in an experimental mouse model of the metabolic syndrome. Cardiovasc Ther 2013; 31: 280-284.

16. Hara T, Fukuda D, Tanaka K, Higashikuni Y, Hirata Y, Nishimoto $\mathrm{S}$, et al. Rivaroxaban, a novel oral anticoagulant, attenuates atherosclerotic plaque progression and destabilization in ApoEdeficient mice. Atherosclerosis 2015; 242: 639-646.

17. He RQ, Tang XF, Zhang BL, Li XD, Hong MN, Chen QZ, et al. Protease-activated receptor 1 and 2 contribute to angiotensin II-induced activation of adventitial fibroblasts from rat aorta. Biochem Biophys Res Commun 2016; 473: 517-523.

18. Kitasato L, Yamaoka-Tojo M, Hashikata T, Ishii S, Kameda R, Shimohama T, et al. Factor Xa in mouse fibroblasts may induce fibrosis more than thrombin. Int Heart J 2014; 55: 357-361.

19. Chan MY, Lin M, Lucas J, Moseley A, Thompson JW, Cyr D, et al. Plasma proteomics of patients with non-valvular atrial fibrillation on chronic anti-coagulation with warfarin or a direct factor Xa inhibitor. Thromb Haemost 2012; 108: 1180-1191.

20. Katoh H, Nozue T, Michishita I. Anti-inflammatory effect of factor-Xa inhibitors in Japanese patients with atrial fibrillation. Heart Vessels 2017; 32: 1130-1136.

21. Bukowska A, Zacharias I, Weinert S, Skopp K, Hartmann C, Huth C, et al. Coagulation factor Xa induces an inflammatory signalling by activation of protease-activated receptors in human atrial tissue. Eur J Pharmacol 2013; 718: 114-123.

22. Kondo H, Abe I, Fukui A, Saito S, Miyoshi M, Aoki K, et al. Possible role of rivaroxaban in attenuating pressure-overloadinduced atrial fibrosis and fibrillation. $J$ Cardiol 2018; 71:310-319.

23. Chung CC, Lin YK, Chen YC, Kao YH, Yeh YH, Chen YJ. Factor $\mathrm{Xa}$ inhibition by rivaroxaban regulates fibrogenesis in human atrial fibroblasts with modulation of nitric oxide synthesis and calcium homeostasis. J Mol Cell Cardiol 2018; 123: 128-138.

24. Imano H, Kato R, Tanikawa S, Yoshimura F, Nomura A, Ijiri $\mathrm{Y}$, et al. Factor Xa inhibition by rivaroxaban attenuates cardiac 
remodeling due to intermittent hypoxia. J Pharmacol Sci 2018; 137: $274-282$.

25. Robin J, Kharbanda R, Mclean P, Campbell R, Vallance P. Protease-activated receptor 2-mediated vasodilatation in humans in vivo: Role of nitric oxide and prostanoids. Circulation 2003; 107: $954-959$.

26. McGuire JJ, Hollenberg MD, Andrade-Gordon P, Triggle CR Multiple mechanisms of vascular smooth muscle relaxation by the activation of proteinase-activated receptor 2 in mouse mesenteric arterioles. Br J Pharmacol 2002; 135: 155-169.

27. Napoli C, De Nigris F, Cicala C, Wallace JL, Caliendo G, Condorelli $\mathrm{M}$, et al. Protease-activated receptor-2 activation improves efficiency of experimental ischemic preconditioning. Am J Physiol Heart Circ Physiol 2002; 282: H2004-H2010.

28. Hamilton JR, Frauman AG, Cocks TM. Increased expression of protease-activated receptor-2 (PAR2) and PAR4 in human coronary artery by inflammatory stimuli unveils endothelium-dependent relaxations to PAR2 and PAR4 agonists. Circ Res 2001; 89: 92-98.

29. Hirota CL, Moreau F, Iablokov V, Dicay M, Renaux B, Hollenberg MD, et al. Epidermal growth factor receptor transactivation is required for proteinase-activated receptor-2-induced COX-2 expression in intestinal epithelial cells. Am J Physiol Gastrointest Liver Physiol 2012; 303: G111-G119.

30. Campbell DJ, Duncan AM, Kladis A, Harrap SB. Angiotensin peptides in spontaneously hypertensive and normotensive Donryu rats. Hypertension 1995; 25: $928-934$.

31. Sano H, Okamoto H, Kitabatake A, Iizuka K, Murakami T, Kawaguchi H. Increased mRNA expression of cardiac reninangiotensin system and collagen synthesis in spontaneously hypertensive rats. Mol Cell Biochem 1998; 178: 51-58.

32. Nakashima $H$, Kumagai $K$, Urata $H$, Gondo $N$, Ideishi $M$, Arakawa K. Angiotensin II antagonist prevents electrical remodeling in atrial fibrillation. Circulation 2000; 101: 2612-2617.

33. Kumagai K, Nakashima H, Urata H, Gondo N, Arakawa K, Saku K. Effects of angiotensin II type 1 receptor antagonist on electrical and structural remodeling in atrial fibrillation. $J \mathrm{Am}$ Coll Cardiol 2003; 41: 2197-2204.

34. Parsons C, Patel SI, Cha S, Shen WK, Desai S, Chamberlain AM, et al. CHA2DS2-VASc score: A predictor of thromboembolic events and mortality in patients with an implantable monitoring device without atrial fibrillation. Mayo Clin Proc 2017; 92: $360-369$.

35. Morillas P, Pallarés V, Fácila L, Llisterri JL, Sebastián ME, Gómez M, et al. The CHADS2 score to predict stroke risk in the absence of atrial fibrillation in hypertensive patients aged 65 years or older. Rev Esp Cardiol (Engl Ed) 2015; 68: 485-491.

36. Welles CC, Whooley MA, Na B, Ganz P, Schiller NB, Turakhia MP. The CHADS2 score predicts ischemic stroke in the absence of atrial fibrillation among subjects with coronary heart disease: Data from the Heart and Soul Study. Am Heart J 2011; 162: $555-561$.

37. Saliba W, Gronich N, Barnett-Griness O, Rennert G. Usefulness of CHADS2 and CHA2DS2-VASc scores in the prediction of new-onset atrial fibrillation: A population-based study. Am $J$ Med 2016; 129: 843-849.

38. Yang Y, Zhang Z, Ng CY, Li G, Liu T. Meta-analysis of CHADS2 score in predicting atrial fibrillation. Am J Cardiol 2015; 116: 554-562.

39. Antoniak S, Cardenas JC, Buczek LJ, Church FC, Mackman N, Pawlinski R. Protease-activated receptor 1 contributes to angiotensin II-induced cardiovascular remodeling and inflammation. Cardiology 2017; 136: 258-268.

40. Grimsey NJ, Trejo J. Integration of endothelial protease-activated receptor-1 inflammatory signaling by ubiquitin. Curr Opin Hematol 2016; 23: 274-279.

\section{Supplementary Files}

Please find supplementary file(s);

http://dx.doi.org/10.1253/circj.CJ-20-1006 